American Journal of Agricultural and Biological Sciences 4 (2): 152-155, 2009

ISSN 1557-4989

(C) 2009 Science Publications

\title{
A Quick Method for Metarhizium anisopliae Isolation from Cultural Soils
}

\author{
${ }^{1}$ M.A. Tajick Ghanbary, ${ }^{2}$ A. Asgharzadeh, ${ }^{1}$ A.R. Hadizadeh and ${ }^{1}$ M. Mohammadi Sharif \\ ${ }^{1}$ Department of Plant Protection, Faculty of Agronomic Sciences, \\ Sari Agricultural Sciences and Natural Resources University, Sari, Iran \\ ${ }^{2}$ Department of Soil Biology, Soil and Water Research Institute, Tehran, Iran
}

\begin{abstract}
Problem statement: Fungi are one of the most active members in biological community of cultural soils. Many saprophyte and facultative parasitic fungi live in soil. Metarhizium anisopliae, one of the most famous soil inhabitant entomopathogens has a virulence potential on plant and animal pests. Approach: Introducing a new method for its isolation from soil was an applied method to find it without any limitation. Metarhizium anisopliae shifts to saprophytic phase and remain alive within soil in absence of susceptible host. As a shortcut, we can transfer the fungus from soil to lab by culturing soil suspension. One hundred cultural soil samples from different regions of Iran were tested to finding Metarhizium isolates. Culturing 1:5000-1:10000 soil suspension on artificial medium containing necessary macro and micronutrients for fungal growth were resulted in isolation. Metarhizium anisopliae isolates were harvested seven days after culturing the suspensions. All isolates were inoculated in $50 \mathrm{~mL}$ PDB in destruxin production assay and 7 days later broth medium was filtrated by using filter paper. Culture filtrates were extracted and in bioassays they were sprayed on larva of citrus leaf miner. Results: Nine isolates of Metarhizium anisopliae were harvested. Microscopic studies showed that morphological features had complete coincidence with valid descriptions of the fungus. Bioassay confirmed that all harvested isolates secrete active and effective destruxin in broth. Conclusion: Isolation of Metarhizium by culturing the soil suspension, a useful method for more studies of the entomopathogen at different geographical regions. Native populations of this fungus had special importance in local biological control programs. This procedure was a costsand time-effective method for pathogen isolation.
\end{abstract}

Key words: Metarhizium anisopliae, Destruxin, Entomopathogenic fungi, Soil

\section{INTRODUCTION}

Nowadays biological control as a practical science is very appreciated and as a solvent for long term usage of chemical pesticides problem is completely notified. There has been an increasing interest in employing fungal pathogens to combat insect pests. New application and production combined with a greater understanding of both fungal and insect ecology have shown that biological insecticides can now compete traditional chemical pesticides much faster. Metarhizium anisopliae the agent of green muscardin disease of insects is an important fungus in biological control of insect pests ${ }^{[6]}$. It is a Deutromycete belonging to Hyphomycetes. The fungus is a facultative parasite which as an entomopathogen can affect a group of insects. There are many similar entomopathogens in nature that live in different ecological environments.
The exact number of entomopathogenic genera and species is indefinite but in some reviews about 90 genera and 700 belonging species were reported ${ }^{[17]}$. Some are obligate parasites and others facultative. The obligates are living on special insects and their laboratory studies is only possible on naturally infected hosts. But facultative have at least two positive scores: (1) Easy in vitro studies and assays, (2) Saprophytic life in absence of suitable host in nature. Metarhizium is an interesting organism that further more direct attacking its host, produces a series of biological active metabolites in vitro and in situ ${ }^{[18]}$. A group of destruxins were identified and purified in most species specially $M$. anisopliae. Other species and varieties have similar biologically active metabolites too. Some important biopesticides are produced as different names in the world from selected Metarhizium isolates. In spite of hundreds of reports about M. anisopliae and its

Corresponding Author: M.A. Tajick Ghanbary, Department of Plant Protection, Faculty of Agronomic Sciences,

Sari Agricultural Sciences and Natural Resources University Sari, Iran

Tell: +989112546616 Fax: +98 1513822578 
features, the researches are insufficient and more and more articles are published monthly. In this article a new and quick method for isolating Metarhizium is introduced which can serve as a shortcut for quick preparation of local populations of the genus for different studies.

\section{MATERIALS AND METHODS}

Soil sampling and fungal isolation: A total of 100 sample each containing $1 \mathrm{~kg}$ cultural soil in depth of $0-20 \mathrm{~cm}$ from different provinces in Iran were collected. The samples in plastic bags were stored at $4^{\circ} \mathrm{C}$ until culture. For isolation $10 \mathrm{~g}$ of each soil sample was subjected to a 1:5000-1:10000 soil suspension and one $\mathrm{ml}$ of final solute was transferred to steril $9 \mathrm{~cm}$ petri plates then culture medium containing $0.5 \mathrm{~g}$ $\mathrm{KH}_{2} \mathrm{PO}_{4}, 0.5 \mathrm{~g} \mathrm{~K}_{2} \mathrm{HPO}_{4}, 0.5 \mathrm{~g}$ peptone, $0.5 \mathrm{~g} \mathrm{MgSO}_{4}$, $10 \mathrm{~g}$ dextrose, $0.5 \mathrm{~g}$ yeast extract, $0.05 \mathrm{~g}$ rosebengal, $0.03 \mathrm{~g}$ streptomycin sulphate. The rose-bengal and streptomycin were added to medium after sterilization before transferring to petries. The isolates were purified by single spore method.

Identification and storage: All morphological features of isolated Metarrhizia were compared with valid descriptions of its different species. For long term storage of collected Metarrhizia, colonies transferred to PDA slants and stored at $4^{\circ} \mathrm{C}$.

Destruxin production tests and bioassay: For this purpose the fungus was inoculated in $50 \mathrm{~mL}$ PDB (potato dextrose broth) in $250 \mathrm{~mL}$ erlenmeyers for one week at room temperature. The broth filtrated from pelletes via whattman No. 1 and culture filtrates subjected to secreted products extraction. For destruxins extraction culture filtrates were mixed with $10 \mathrm{~mL}$ chloroform and shake vigorously for $10 \mathrm{~min}$. After an hour chloroform was separated from broth and completely evaporated. The residue was resolved in $10 \mathrm{~mL}$ distilled water and stored at $-20^{\circ} \mathrm{C}$ until application. Fresh infected different citrus leaves to citrus leaf miner from gardens were prepared for bioassay in $9 \mathrm{~cm}$ petri plates. The crude extract and its dilutions were sprayed on leaves then treatments were observed daily on a stereomicroscope.

\section{RESULTS}

After 3-5 days different fungal colonies grew and sporulation completed about 7-10 days. In primary investigations on different fungal colonies via a stereomicroscope and morphological comparisons suspected Metarhizium colonies were selected and transferred to PDA slants. Culture of soil samples lead to nine Metarhizium isolates. Species identification process showed that all belong to M. anisopliae with small differences. Most of morphological features had high coincidence with species description. Fungal storage at $4^{\circ} \mathrm{C}$ remained them alive at least for 6 months. All isolates produced total destroxins without shaking at room temperatures in PDB medium after one week. The isolates sporulated easily on PDA and PDB. There was no difference between isolates for destruxin production, in a test some two weeks old cultures proceeded for toxins extraction, total destruxins remained stable at least for two weeks at room temperature. The crude extracts of total destroxins and its serial diluted fractions up to seventh dilution had total mortality on different larval stages on all citrus varieties. Larval mortality started on second day after treatments regardless to their stages. In another test no difference was observed in effects of $-20^{\circ} \mathrm{C}$ stored and fresh extracts on larvae of citrus leaf miner.

\section{DISCUSSION}

In modern and sustainable agriculture chemical pesticides are exchanged for alternative strategies of pests control. Entomopathogenic fungi showed high performance in integrated pest management programs that resulted meaningful decrease in using insecticides. Under natural conditions entomopathogenic fungi are the most important mortality factor of natural insect populations and is safe for non target organisms ${ }^{[22]}$. Potentially all insect groups may be affected by more than 700 species of entomopathogenic fungi ${ }^{[15]}$. Some are facultative parasites (Fusarium or Aspergillus) and others obligate parasites (Entomophthora), some are completely host specific (Cordyceps). The Entomophthorales are so effective on aphids and flies in humid and warm regions,but attempts for preparing commercial mycoinsecticides of them failed, because their mass production is only possible on live hosts, furthermore their favorite conditions is not always present in the fields. For this reason most attemps are focused on mitosporic easy amplifable fungi like Metarhizium, Beauveria, Verticillium and Paecilomyces. Most have a wide host range, some very high intraspecefic genetic variation, many have exact host specifity. In contrast to other biological control agents fungi can penetrate directly from host cuticle and have no necessity for entrance in its digestive system, then can affect sucker insects. Metarhizium anisopliaeis is a worldwide and interesting soil inhabiting entomopathogen. Its first description was written by 
Russian mycologist Metschnikoff in 1879 as an Entomophthora species E. anisopliae, then was corrected by Sorokin in 1883 to M. anisopliae. During the last 50 years Metarhizium and allied species specially $M$. anisopliae absorbed the attention of many scientists as mycologists, entomologists, biologists and so on. The most investigated aspect of Metarhizium is its application as biological control agent of plants and domestic animals pests. Some examples of successful applications are control of termits ${ }^{[24]}$, onion thrips ${ }^{[13]}$, tobbaco whitefly, red spider mite ${ }^{[4]}$, eggs of mites ${ }^{[21,25]}$, fruit flies, mosquitoes ${ }^{[1]}$, green leafhopper ${ }^{[23]}$, rice wevill $^{[5]}$, flour bettle ${ }^{[23]}$,some animal arthropods ${ }^{[7]}$, vein vewil $^{[20]}$, ectoparasitic animal mites ${ }^{[8]}$ and more other examples were listed by Bruck ${ }^{[9]}$. Furthermore above research reports, various bioinsecticides against a range of insect pests were released as commercial products $^{[3,10,14]}$. In Bruck's belief ${ }^{[9]}$ success in application of biological control agents in nature has close relationship with their related biological systems specially their ecological niche. The subject is exactly true for entomopathogenic fungi. Our information about entomopathogens biology far from their host body is insufficient. A special pathogen may be present and active in an environment then infect insect pest after its entrance to that region. An example is the control of black vine weevil by Metarhizium anisopliae ${ }^{[9]}$. Pathogenicity and virulence variation between different species, isolates and situations were discussed by ${ }^{[19]}$. Based on another report produced inocula on naturally infected insects is more virulent and effective than harvested inoculums from axenic culture media on sensitive insect hosts ${ }^{[2]}$. This is important specially for mass production of Metarhizium and other entomogens which are using as mycoinsecticides. A suspected reason for decrease in pathogen virulence is ingredients of culture media like carbohydrates ${ }^{[12,19]}$. Metarhizium is one of the most promising biological controlling agents against insect pests specially some soil inhabitants like scarab grubs ${ }^{[11,16]}$. There are many attempts for testing some Metarhizium formulations and application methods on soil insects. Metarhizium anisopliae has been isolated from infected hosts in nature. Collecting native isolates of each geographical region is possible via field search and finding naturally infected dead insects. This method is very time consuming and season restricted, because the environmental factors for infection of live hosts aren't present during the year. The introduced method by this research without no restrictions is executable for ever and cause saving time and costs for all local studies on Metarhizium anisopliae biology, populations biology, pathogenicity, viability in soil, variations in total destruxins production, ecology and many other purposes.

\section{CONCLUSION}

Culture of soil suspension in artificial media has a good chance to find Metarhizium in soil. The entomopathogen finding in local regions without susceptible bait is nearly impossible and this method can serve as a shortcut without any host, time and season limitations. Complementary approaches after taxonomic identification like bioassay confirmes the isolation results. Local populations of Metarhizium anisopliae have special role in integrated pest management programs at different regions.

\section{REFERENCES}

1. Alves, S.B., L.F. Alves, R.B. Lopes, R.M. Pereira and S.A. Viera, 2002. Potential of some Metarhizium anisopliae isolates for control of Culex quinquefasciatus (Dipt., Culicidae). J. Applied Entomol., 126: 504-509. DOI: 10.1046/j.1439- 0418.2002.00674.x

2. Arzumanov, T., N. Jenkins and S. Roussos, 2005. Effect of aeration and substrate moisture content on sporulation of Metarhizium anisopliae var. Acridum. Process Biochem., 40: 1037-1042. DOI: 10.1016/j.procbio.2004.03.013

3. Michalaki, M.P., C.G. Athanassiou, N.G. Kavallieratos and Y.A. Batta et al., 2005. Effect of soil application of different formulations of Metarhizium anisopliae on African tephritid fruit flies and their associated endoparasitoids. Biol. Control., 35: 83-91. DOI: 10.1016/j.cropro.2005.07.003

4. Batta, Y.A., 2003. Production and testing of novel formulation of the entomopathogenic fungus Metarhizium anisopliae (metschnikoff) Sorokin (deuteromycotina: Hyphomycetes). Crop Protect., 22: 415-422. DOI: 10.1016/S0261-2194(02)00200-4

5. Batta, Y.A., 2004. Control of rice weevil (Sitophilus oryzae L., coleoptera: Curculionidae) with various formulations of Metarhizium anisopliae. Crop Protect., 23: 103-108. DOI: 10.1016/j.cropro.2003.07.001

6. Bhanu Prakash, G.V.S., V. Padmaja and R.R. Siva Kiran, 2008. Statistical optimization of process variables for the large-scale production of Metarhizium anisopliae conidiospores in solid-state fermentation. Biores. Technol., 99: 1530-1537. DOI: 10.1016/j.biortech.2007.04.031 
7. Briggs,L.L., D.D. Colwel and R. Wall, 2006. Control of the cattle louse Bovicola bovis with the fungal pathogen Metarhizium anisopliae. Vet. Parasitol., $\quad$ 142: 344-349. doi:10.1016/j.vetpar.2006.07.018

8. Brooks, A. and R. Wall, 2005. Horizontal transmission of fungal infection by Metarhizium anisopliae in parasitic psoroptes mites (acari: Psoroptidae). Biol. Control, 34: 58-65. $\quad$ DOI: 10.1016/j.biocontrol.2005.03.016

9. Bruck, D.J., 2004. Ecology of Metarhizium anisopliae in soilless potting media and the Rhizosphere: Implications for pest management. Biol. Control, 32: 155-163. DOI: 10.1016/j.biocontrol.2004.09.003

10. Butt, M. and G. Leonard, 2000. Fungal biological control agents. Pestic. Outlook, 11: 186-191. DOI: $10.1039 /$ b008009h

11. Hiromori, H. and J. Nishigaki, 2001. Factor analysis of synergistic effect between the entomopathogenic fungus Metarhizium anisopliae and synthetic insecticides. Applied Entomol. Zool., 36: 231-236.

http://www.jstage.jst.go.jp/article/aez/36/2/231/pdf

12. Lee, S., H. Kinoshita, F. Ihara, Y. Igarashi and T. Nihira, 2008. Identification of novel derivative of helvolic acid from Metarhizium anisopliae grown in medium with insect component. J. Biosci. Bioeng., 105: 476-480. DOI: 10.1263/jbb. 105.476

13. Maniania, N.K., S. Sithanantham, S. Ekesi, K. Ampong-Nyarko, J. Baumgartner, B. Lohr and C.M. Matoka, 2003. A field trial of the entomopathogenic fungus Metarhizium anisopliae for control of onion thrips, Thrips tabaci. Crop Protect., 22: 553-559. DOI: 10.1016/S0261-2194(02)00221-1

14. Ekesi, N.K. Maniania, S.A Mohamed and S.A. Lux, 2005. Effect of soil application of different formulations of Metarhizium anisopliae on African tephritid fruit flies and their associated endoparasitoids. Biol. Control, 35: 83-91. DOI: 10.1016/j.biocontrol.2005.06.010

15. Milner, R.J., 2000. Current status of Metarhizium as a mycoinsecticide in Australia. Biocontrol News Infom., 21: 47-50. http://www.pestscience.com/PDF/BNIra53.PDF

16. Mochi, D.A., A.C. Monteiro, J.C. Barbosa, 2008. Action of pesticides to Metarhizium anisopliae in soil. Neotropic. Entomol., 34: 961-971. DOI: 10.1590/S1519-566X2005000600013

17. Onofre, S.B., C.M. Miniuk, N.M. Barros and J.L. Azevedo, 2001. Growth and sporulation of Metarhizium flavoviride var. Flavoviride on culture media and lighting regimes. Scientia Agricola, 58: 613-616. DOI: 10.1590/S0103-90162001000300026
18. Rao, Y.K., C.H. Tsou and Y.M. Tzeng, 2006. Antioxidants enhanced production of destruxin E from cultivation of Metarhizium anisopliae. Applied Microbiol. Biotechnol., 73: 519-524. DOI: 10.1007/s00253-006-0493-4

19. Shah, F.A., C.S. Wang and T.M. Butt, 2005. Nutrition influences growth and virulence of the insect-pathogenic fungus Metarhizium anisopliae. FEMS. Microbiol., 251: 259-266. DOI: 10.1016/j.femsle.2005.08.010

20. Shah, F.A., M.A. Ansari, M. Prasad and T.M. Butt, 2007. Evaluation of black vine weevil (Otiorhynchus sulcatus) Control strategies using Metarhizium anisopliae with sublethal doses of insecticides in disparate horticultural growing media. Biol. Control, 40: 246-252. DOI: 10.1016/j.biocontrol.2006.10.005

21. Shi, W.B. and M.G. Feng, 2004. Lethal effect of Beauveria bassiana, Metarhizium anisopliae and Paecilomyces fumosoroseus on the eggs of Tetranychus cinnaharinus (acari: Tetranychidae) with a description of a mite egg bioassay system. Biol. Control, 30: 165-173. DOI: 10.1016/j.biocontrol.2004.01.017

22. Toriello, C., A.P. Torres, A.B. Diaz, H.N. Barranco, A.P. Mejia, M.L. Jimenez and T. Mier, 2006. Lack of acute pathogenicity and toxicity in mice of an isolate of Metarhizium anisopliae var. Anisopliae from spittlebugs. Ecotoxicol. Environ. Safe., 65: 278-287. DOI: 10.1016/j.ecoenv.2005.07.016

23. Tounou, K., K. Agboka, H. Poehling, K. Raupach, J. Langewald, G. Zimmermann and C. Orgemeister, 2003. Evaluation of the entomopatogenic fungi Metarhizium anisopliae and Paecilomyces fumosoroseus (deuteromycotina: Hyphomycetes) for control of the green leafhopper Empoasca decipiens (homoptera: Cicadellidae) and potential side effects on the egg parasitoid Anagrus atomus (hymenoptera: Mymaridae). Biocontrol Sci. Technol., 13: $\quad 715-728 . \quad$ DOI: 10.1080/09583150310001606534

24. Wang, C. and J.E. Powell, 2004. Cellulose bait improves the effectiveness of termites (isoptera: Rhinotermitidae). Biol. Control, 30: 523-529. DOI: 10.1016/j.biocontrol.2004.02.007

25. Wekesa, V.W., M. Knapp, N.K. Maniania and H.I. Boga, 2006. Effects of Beauveria bassiana and Metarhizium anisopliae on mortality, fecundity and egg fertility of Tetranychus evansi. J. Applied Entomol., 130: 155-159. DOI: 10.1111/j.14390418.2006.01043.x 